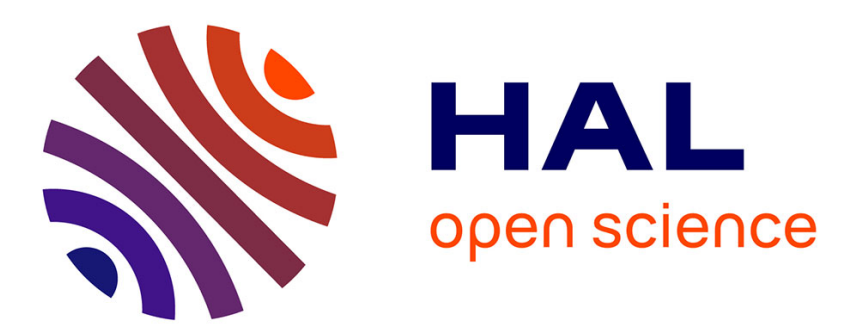

\title{
Identifying representative muscle synergies in overhead football throws
}

Ana Lucia Cruz Ruiz, Charles Pontonnier, Anthony Sorel, Georges Dumont

\section{To cite this version:}

Ana Lucia Cruz Ruiz, Charles Pontonnier, Anthony Sorel, Georges Dumont. Identifying representative muscle synergies in overhead football throws. Computer Methods in Biomechanics and Biomedical Engineering, 2015, pp.2. 10.1080/10255842.2015.1070581 . hal-01174114

\author{
HAL Id: hal-01174114 \\ https://hal.inria.fr/hal-01174114
}

Submitted on 8 Jul 2015

HAL is a multi-disciplinary open access archive for the deposit and dissemination of scientific research documents, whether they are published or not. The documents may come from teaching and research institutions in France or abroad, or from public or private research centers.
L'archive ouverte pluridisciplinaire HAL, est destinée au dépôt et à la diffusion de documents scientifiques de niveau recherche, publiés ou non, émanant des établissements d'enseignement et de recherche français ou étrangers, des laboratoires publics ou privés. 


\title{
Identifying representative muscle synergies in overhead football throws
}

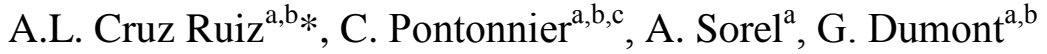 \\ ${ }^{a}$ IRISA/INRIA MimeTIC, Rennes, France; ${ }^{b}$ ENS Rennes, Bruz, France; ${ }^{c}$ Ecoles de Saint-Cyr Coëtquidan, Guer, France
}

Keywords: motor control; synchronous synergies; EMG; matrix factorization

\section{Introduction}

Human motion control requires the command of a highly redundant and nonlinear musculoskeletal system. This complex motor control problem has been simplified through the theory of muscle synergies. According to this theory, the control of motion by the CNS (central nervous system) is made by translating task-level commands into a reduced number of modules or synergies (Muceli et al. 2010).

In the current abstract we show the existence of such synergies for a group of dynamic motions of the upper-body: overhead football throws. The purpose of this study was to find a compact control representation of throwing for the future design of efficient motion controllers for avatar animation. The controllers will adapt this representation allowing muscle-actuated avatars to reproduce the recorded and new throwing motions.

\section{Methods}

\subsection{Data collection and processing}

The motion consisted in a right-hand throw with an American football to a $4 \mathrm{~m}$ target (a description of this motion is featured in Fig.1(top)). The muscle activity of 16 right arm and trunk muscles was collected from a healthy 32-year old male (stature, $1.86 \mathrm{~m}$, weight 72 $\mathrm{kg}$ ), using surface electrodes (Cometa Waveplus EMG system). The EMGs were amplified (gain $1000)$, digitized $(1 \mathrm{kHz})$, band-pass filtered (10$450 \mathrm{~Hz})$, rectified, and low-pass filtered $(6 \mathrm{~Hz})$. ECG artifacts were removed using an ICA-based filtering procedure. Motion was captured with a Vicon system (15 cameras, $100 \mathrm{~Hz}$ sampling rate).

\subsection{Synchronous synergy model}

A synchronous synergy $w_{i}$ is defined as a $D$ dimensional vector of coefficients, capturing a specific relationship in the strength of activation of $D$ muscles. Each synergy is paired with a time-varying combination coefficient vector $\left(c_{i}\right)$, which determines its temporal evolution. A set of $N$-synergies can be linearly combined to generate $D$-muscle patterns $M(t)$ :

$M(t)=W C(t)=\sum_{i=1}^{N} w_{i} c_{i}(t)$

Where, $M(t)$ is the $D \times T_{\text {samples }}$ matrix containing the recorded muscle patterns, $W$ is the $D \times N$ muscle synergy matrix, and $C(t)$ is the $N \times T_{\text {samples }}$ combination coefficient matrix.

In our case matrix $M(t)$ was created by concatenating the muscle data for 8 throws.

\subsection{Synergy extraction}

A NMF (Non-negative matrix factorization) algorithm (Kim \& Park 2008) was used to extract a set of muscle synergies and their corresponding combination coefficients from the recorded EMG patterns. Essentially, NMF decomposes a nonnegative matrix into a non-negative linear combination of basis vectors, by solving the following optimization problem,

$$
\min _{\mathrm{W}, \mathrm{C}} \frac{1}{2}\|M(t)-W C(t)\|_{F}^{2} \text {, subject to } W, C(t) \geq 0
$$

\subsection{Synergy model order}

The criterion for determining the synergy model order was based on the average coefficient of determination $\left(r^{2}\right)$ between the original and reconstructed muscle patterns (d'Avella, et al. 2003; Muceli et al. 2010). The chosen number of synergies corresponded to the sharpest change in the slope of the $r^{2}$ curve. For throwing, the sharpest change occurred at 3 synergies $\left(r^{2}=0.7352\right)$.

\section{Results and discussion}

The extracted synergies were used to reconstruct the 16 original muscle patterns of the right arm and trunk, during an overhead football throw using Equation (1). The performed motion and example reconstructions are featured in Fig.1. 
The reconstruction performance increases with the number of synergies, however after 3 synergies there is no abrupt change in performance.

The extracted synergies and their combination coefficients are featured in Fig.2. Each synergy recruits groups of muscles with similar biomechanical action, and corresponds to specific motion phases.

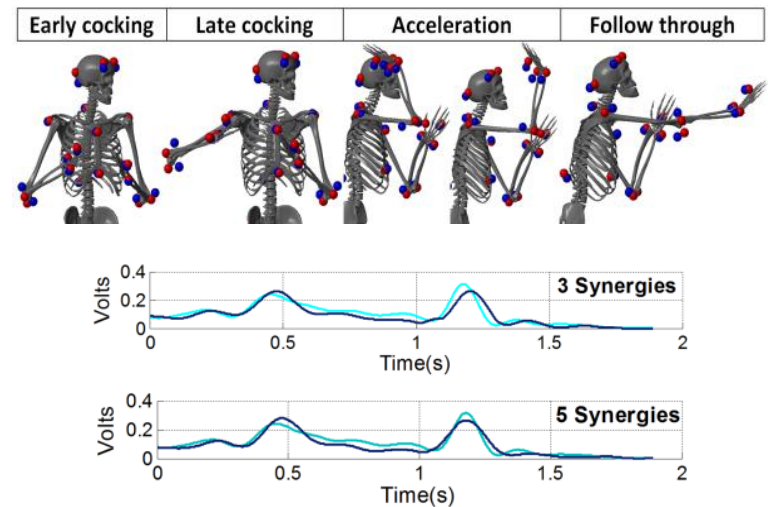

Figure 1 Motion phases \& wrist extensor group excitation (blue-original, dark blue-reconstructed)

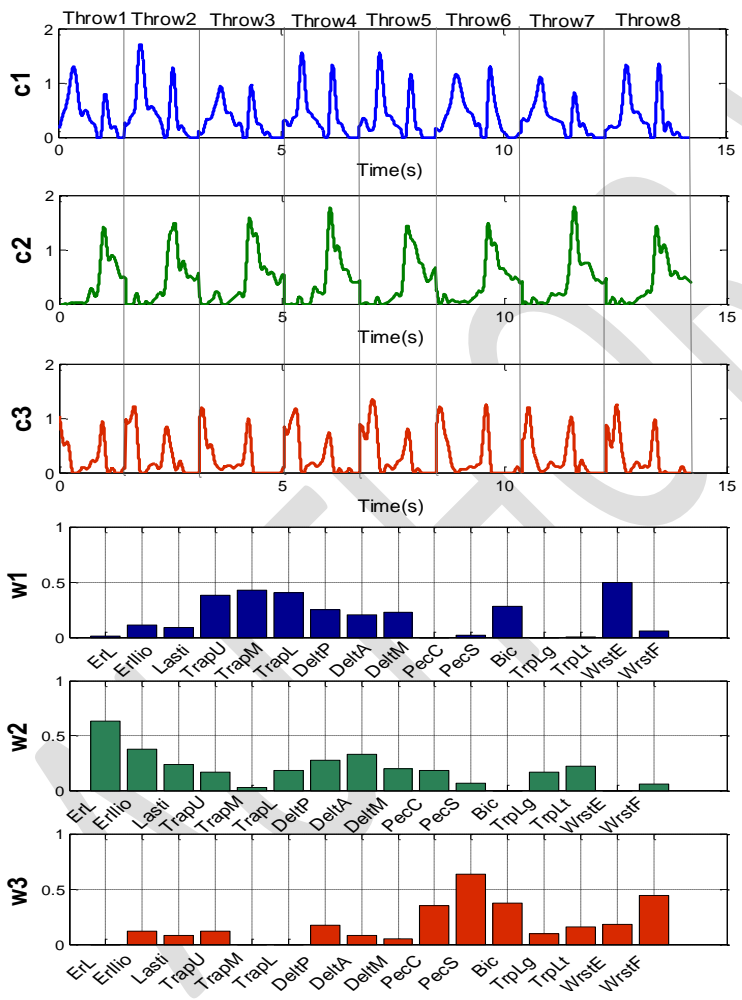

Figure 2 Combination coefficients and synergies

Synergy $w_{1}$, contains the biomechanical actions needed during the late cocking phase. Therefore, the most active muscles correspond to actions such as: shoulder abduction, external rotation and scapular retraction. This synergy was also active during the follow through phase, as a decelerator. Synergy $w_{2}$, comprises actions necessary during the acceleration phase, such as: trunk extension, rotation and elbow extension. Finally, synergy $w_{3}$ includes actions that contribute both to the early cocking phase and the acceleration phase, such as shoulder internal rotation, elbow and wrist flexion.

We ranked these 3 synergies according to their degree of contribution or power contained in the functions $w_{i} c_{i}(t)$ (in the time domain). The synergy displaying the highest power was synergy $w_{2}$, followed by synergies $w_{1}$, and $w_{3}$. These results are consistent with the expectation that power is highest during the acceleration phase. We also observed that the synergies themselves, their triggering order, and degree of contribution were preserved in higher order synergy models and in models derived from single throws.

\section{Conclusions}

We have evidenced the presence of synchronous muscle synergies in a throwing motion and analysed their relationship with biomechanical outputs. The results also evidence the robustness and repeatability of this components across different throws.

Our future work, includes designing a more generic synergy model for throwing by including throws to $2 \mathrm{~m}$ and $7 \mathrm{~m}$ in the NMF decomposition. We also plan to validate further the optimal synergy model order through a more robust measure of reconstruction performance (ex. coefficient of determination within a cross-validation procedure). Our final goal will be to use the extracted components for the construction of muscle-based controllers for avatar animation.

\section{Acknowledgements}

This work is supported by the French ANR project ENTRACTE (Grant agreement: ANR 13-CORD002-01).

\section{References}

D'Avella A, Saltiel P, Bizzi E. 2003. Combinations of muscle synergies in the construction of a natural motor behavior. Nat. Neuroscience. 6(3): 300308.

Kim H, Park H. 2008. Nonnegative matrix factorization based on alternating nonnegativity constrained least squares and active set method. SIAM J. Matrix Anal. Appl. 30(2): 713-730.

Muceli S, Boye AT, d'Avella A, Farina D. 2010. Identifying representative synergy matrices for describing muscular activation patterns during multidirectional reaching in the horizontal plane. J. Neurophysiol. 103(3): 1532-1542. 